\title{
A Study on the Use of Chinese Degree Complement of Foreign Students
} Cai Li

\author{
College of Chinese Language and Culture, Jinan University, Guangzhou, Guangdong,China \\ caili@hwy.jnu.edu.cn
}

Keywords: Degree complement, Use, Foreign students, corpus.

\begin{abstract}
In this paper, this author takes the 43 degree complements in modern Chinese as retrieval terms, to retrieve HSK corpus of Beijing Language and Culture University and corpus of Southeast Asian students of Chinese Studies at Jinan University. On the basis of these data,the article investigates the mastery and use of Chinese degree complement by foreign students, and targeted analysis of typical errors is made also.
\end{abstract}

\section{外国留学生使用汉语程度补语的情况研究}

\author{
蔡丽 \\ 暨南大学华文学院, 广州, 广东, 中国 \\ caili@hwy.jnu.edu.cn
}

关键词：程度补语; 使用; 留学生; 语料库

中文摘要. 本文分别以现代汉语中 43 个程度补语为检索项检索北京语言大学HSK作文语料库 和暨南大学华文学院东南亚留学生作文语料库, 全面考察留学生对汉语程度补语的掌握与使 用情况, 并对典型偏误进行了针对性分析。

\section{1. 引言}

在现代汉语中, 程度补语同程度副词一样, 是一个相对封闭的类, 程度补语存在一系列 有别于其他类型补语的句法语义特征。Cai Li（2011）全面分析了程度补语的立类依据, 并依 此标准确定现代汉语中程度补语的43个成员, 并进行了多角度的分类。Cai Li (2012) 采用计 算语言学的自动分词技术对北京大学现代汉语语料库中检索到的全部相关语料进行计算与分 析, 在大规模语料统计结果的基础上, 考察、分析 43 个程度补语与充当述语的词语的组合特 征, 总结其中的规律。本文在此研究基础上, 分别以 43 个程度补语为检索项检索北京语言大 学HSK作文语料库和暨南大学华文学院东南亚留学生作文语料库, 以考察外国留学生对汉语 程度补语的掌握与使用情况。

\section{2. 留学生对程度补语的使用率分析}

\section{1 程度补语在HSK作文语料库中出现情况分析}

在HSK作文语料库中检索到的程度补语共有 25 个，占 $58.14 \%$ ，包括“多、极、很、不堪、 万分、无比、坏、透、不过、厉害、死、要命、不得了、慌、可怜、之至、要死、透顶、不 行、疯、过分、异常、够呛、绝顶、绝伦”。 
在HSK作文语料库中未检索到的程度补语共有 18 个，占 $61.86 \%$ ，包括“邪行、不成、够受 的、够劲、够燋的、邪乎、蒙、离谱、去了、凶、什么似的、了不得、可以、俊、非常、吓 人、惊人、出奇”。

\section{2 程度补语在东南亚留学生作文语料库中出现情况分析}

在东南亚留学生作文语料库中检索到的程度补语共有 22 个, 占 $51.16 \%$, 包括“不堪、万分、 不过、不得了、不行、多、过分、很、慌、可怜、厉害、了不得、要命、要死、疯、透顶、 透、无比、之至、坏、极、死”。

在东南亚留学生作文语料库中未检索到的程度补语共有 21 个, 占 $48.84 \%$, 包括“去了、够 呛、够受的、邪乎、吓人、够燋的、凶、邪行、够劲、惊人、出奇、离谱、绝顶、绝伦、什 么似的、异常、不成、可以、非常、蒙了、俊了”。

\section{3 程度补语在两个中介语语料库中出现情况的对比分析}

在两个留学生汉语中介语语料库中均未出现的程度补语共 17 个, 包括“出奇、惊人、吓人、 非常、俊、可以、什么似的、凶、去了、离谱、蒙、邪乎、够燋的、够劲、够受的、不成、 邪行”; 只在HSK作文语料库里出现的有 4 个, 分别是“异常、够呛、绝顶、绝伦”; 只在东南 亚留学生作文语料库中出现的 1 个，即“了不得”。

在两个中介语语料库中均出现了的程度补语共 21 个，包括“多、极、很、不堪、万分、无 比、坏、透、不过、厉害、死、要命、不得了、慌、可怜、之至、要死、透顶、不行、疯、 过分”。其中, “之至、透顶、不行、过分”与程度补语搭配的词语种数仅1次。在两个语料库 中出现次数均高于 1 次的只有“多、极、很、不堪、万分、无比、坏、透、不过、厉害、死、 要命、不得了、慌、要死”等 15 个程度补语; 高于10次的只有“多、极、很、不过、死、要命、 不得了”这7个。

北京大学现代汉语语料库共 2.6 亿字, 总词语次数为 100 次的词语的出现率约为 $0.00039 \%$; HSK 作文语料库的总字数为 424 万, 总词语次数为 17 次的词语的出现率与母语语料库相当; 东 南亚留学生作文语料库的总字数为 400 万, 总词语次数为 16 次的词语的出现率与母语语料库相 当。在母语语料库中, 总词语次数在 100 次以上的程度补语共 30 个; 在HSK作文语料库中, 总 词语次数在 17 次以上的程度补语共 8 个, 分别是“多、很、极、不得了、要命、不过、死、不 堪”; 在东南亚留学生作文语料库中, 总词语次数在 16 次以上的共 9 个, 分别是“多、极、很、 不得了、死、要命、慌、无比、不过”。在两个中介语语料库中出现次数均较高的程度补语共 7 个, 分别是“多、很、极、不得了、要命、死、不过”。

总体而言, 留学生对程度补语使用的词语种数远少于母语使用者, 呈现高频集中使用少 数几个程度补语的特点, 而且程度补语总使用频率普遍低于母语。也就是说, 留学生能掌握 程度补语的基本用法，但掌握的种类有限。

在母语中使用频率不高的程度补语在中介语语料库中也很少出现或不出现, 而在母语中 使用频率高, 但在中介语语料库中却不出现或出现很少的补语主要是以下几类:

第一, 书面化的程度补语。我们考察的两上中介语语料库均为作文语料库, 作文是一种 书面呈现形式, 其中所使用的语言应与一般口语有区别, 出现书面表达方式的频率应更高。 根据第五章的相关分析, 书面语色彩较强的程度补语包括“万分、无比、不堪、异常、之至、 绝伦、透顶、非常、绝顶”等9个。但根据考察发现, 这些书面语色彩较强的程度补语仅有“不 堪、万分、无比、之至、透顶” 5 个在两个中介语语料库中均出现了, 只在HSK作文语料库里 出现的有 3 个, 分别是“异常、绝顶、绝伦”, “非常”在两个语料库中均未出现。其中, 除“无 比、不堪”分别在东南亚留学生作文语料库和HSK作文语料库中出现了一定频次外, 其他的都 仅出现一两次, 与程度补语搭配的词语种数也仅 1 个。整体上看, 留学生对具有书面色彩的程 度补语的使用频率很低。 
第二, 从色彩义来看, 留学生能掌握襄义、中性和贬义三种不同色彩义的程度补语, 但 由于中性和贬义程度补语的成员数量较多, 有选择空间, 留学生在使用时倾向于选择那些由 常用词构成的程度补语。在两个留学生汉语中介语语料库中均未出现的程度补语共 17 个, 其 中中性程度补语有“出奇、惊人、非常、可以、什么似的、去了、够燋的、够劲、不成”9个, 贬义程度补语有“吓人、俊、凶、离谱、蒙、邪乎、邪行、够受的” 8 个, 分别占两类程度补语 总数的 $45 \% 、 42.11 \%$ 。

第三, 从程度的类型来看, 留学生掌握了不同程度等级的程度补语表达方式, 但各类别 都仅掌握其中主要成员的用法。过量级程度补语包括“过分、离谱”两个, 留学生掌握了其中 的“过分”; 顶级程度补语数量众多, 共 31 个, 留学生掌握了其中最常用的19个, 还有相当一 部分, 包括“出奇、惊人、非常、可以、去了、不成、吓人、俊、凶、蒙、邪乎、邪行” 12 个 未使用, 占全部顶级程度补语的 $38.71 \%$; 高级程度补语中有“什么似的、够燋的、够劲、够受 的”4个未使用, 占 $44.44 \%$ 。

\section{3. 留学生对程度补语组合规律掌握情况的分析}

\section{1 对程度补语所搭配词语音节的倾向性选择规律掌握情况分析}

需与双音节词语搭配的程度补语在中介语中出现的有“不堪、万分、无比、异常、之至、 绝伦、透顶、绝顶”, 留学生所使用的与之搭配词语基本为双音节, 仅“无比”出现1例偏误情 况, 如下:

（1）因宿舍每天都有提供三餐, 而且宿舍是新建的, 除了交通有些不便以外, 都很方便 无比。

严格地说, 这例偏误属于程度叠加产生的偏误, 前面已有表示程度的副词“很”修饰, 后面就不能再用程度补语。

\section{2 对程度补语所搭配词语词类的倾向性选择规律掌握情况分析}

部分程度补语对与之搭配的词语的词类有一定的倾向性选择，“万分、什么似的、不得了、 不行”等倾向于与心理、感知动词或形容词组合, “绝伦、绝顶、去了、可怜、惊人、可以、 出奇、吓人、离谱”等倾向于与普通性质形容词组合, 如; “凶”则倾向于与一般动词组合。上 述这些程度补语在中介语语料库出现了的有“万分、了不得、不行、绝伦、绝顶、可怜” 6 个。

其中, 在东南亚留学生作文语料库中出现的有“万分、了不得、不行、可怜”4个，与这几 个程度补语组合的词语分别如下:

（1）万分 (总词种数: 5, 总词语次数：9）激动（4）、伤心（2）、惊喜、感动、高兴

(2) 了不得 (总词种数: 1 , 总词语次数: 1) 高兴

(3) 不行 (总词种数: 1 , 总词语次数: 1 ) 得意

(4) 可怜 (总词种数: 1 , 总词语次数: 3 ) 少（3)

分析上述组合词语, 可以发现, 与“万分、了不得、不行”搭配的均为心理感知动词/形容 词，与“可怜”搭配的均为一般形容词“少”。

在HSK作文语料库中出现的有“万分、不行、可怜、绝顶、绝伦” 5 个, 与这几个程度补语 组合的词语分别如下:

（1）万分 (总词种数: 7, 总词语次数：9）雀跃（2）、高兴（2）、感激、紧张、精 彩、感谢、羡慕

(2) 不行 (总词种数: 1, 总词语次数: 1) 累

（3）绝顶 (总词种数: 1, 总词语次数: 1) 聪明

（4）绝伦 (总词种数: 1, 总词语次数: 1) 精采

(5) 可怜 (总词种数: 1, 总词语次数: 2) 少 (2) 
分析上述组合词语，可以发现，与“万分、不行”搭配的均为心理感知动词/形容词，与“可 怜”搭配的均为一般形容词“少”，与“绝顶、绝伦”搭配的均为一般形容词“聪明、精彩”。

除与“万分”组合的词语种数大于 1 外，其他程度补语的组合词语均为 1 , 因此, 无法据此 判定留学生对程度补语所搭配词语词类的倾向性选择规律掌握情况, 但从中可以发现, 留学 生在使用程度补语时用法集中的特点, 例如, 两个中介语语料库中“可怜”均出现了几次, 但 与之搭配的词语均为“少”, 也就是说学生在习得该用法时有可能将其作为一个整体表达形式 储存。而从“万分”的搭配情况来看, 学生掌握了该程度补语的用法特点, 与之搭配的均为双 音节的心理感知动词/形容词。

\section{3 对程度补语所搭配词语意义的倾向性选择规律掌握情况分析}

我们在考察程度补语的组合情况时发现, 发现有一些程度补语对与之组合的词语的词汇 意义有一定的选择, 并非任何词语都能自由与之搭配。例如:

“万分、什么似的”主要与形容心理感受的词语组合。“离谱”主要与单音节的性质形容词 组合。“绝顶”最常搭配的词语是“聪明”, 共计 72 例, 占全部语料的 $85.71 \%$ 。这是全部程度补 语组合现象中最特别的一个, 即某个词语与某一程度补语搭配频率极高。“绝伦”主要与带有 “精”“美”“荒”“雄”“奇”等义素的词语组合。“可怜”倾向于与指小类的形容词组合。“去了”倾向 于与指大类的形容词组合。“惊人”倾向于与指大类的度量形容词组合。

上述几个程度补语中“万分、绝顶、绝伦、可怜”在中介语语料库中出现了, 下面我们考 察它们在中介语语料库中搭配词语的相关情况。

其中, 在东南亚留学生作文语料库中出现的有“万分、可怜” 2 个, 与这几个程度补语组合 的词语分别如下: 兴

（1）万分（总词种数： 5, 总词语次数：9）激动（4）、伤心（2）、惊喜、感动、高

(2) 可怜 (总词种数: 1 , 总词语次数: 3 ) 少（3）

分析上述组合词语，可以发现，与“万分”搭配的均为形容心理感受的词语，与“可怜”搭 配的均为“少”。

在HSK作文语料库中出现的有“万分、绝顶、绝伦、可怜” 4 个, 与它们组合的词语如下:

（1）万分（总词种数： 7, 总词语次数：9）雀跃（2）、高兴（2）、感激、紧张、精 彩、感谢、羡慕

（2）绝顶（总词种数: 1 , 总词语次数: 1) 聪明

（3）绝伦 (总词种数: 1 , 总词语次数: 1) 精采

（4）可怜（总词种数: 1 , 总词语次数: 2) 少 (2)

分析上述组合词语，可以发现，与“万分”搭配的均为形容心理感受的词语，与“绝顶”搭 配的正是“聪明”，与“绝伦”搭配的是带有“精”的“精采”，与“可怜”搭配的均为“少”。

从上述三大方面综合来看, 留学生对程度补语组合规律特点的掌握情况均呈现出与母语 使用的倾向性规律一致的情况, 但普遍存在掌握的搭配词语偏少的情况, 部分程度补语存在 词块习得的特点, 也就是说将该程度补语和与之搭配的词语作为一个整体习得, 因而用法固 定, 尚未形成灵活运用的能力。

\section{4. 留学生使用程度补语的偏误情况分析}

在整理中介语语料的过程中, 我们发现, 学生在使用程度补语时存在以下主要偏误情况。

\section{1 误加}

4.1.1 程度表达形式叠加

具体表现为同时使用程度副词和程度补语来表达高程度。例如: 
（2）但是我妈妈平常爱吃“绿色食品”，我妈妈是特别瘦极了，她不喜欢吃肉。

例（2）想表达妈妈非常瘦的意思，“瘦”之前用了表示程度高的副词“特别”，之后又用了 程度补语“极”，属于程度表达形式叠加。

\section{1 .2 在粘合式程度补语与组合词语之间插入其他成分}

粘合式程度补语是直接用在述语之后表示程度的补语, 述语和补语紧密粘合, 不能插入 其他成分。例如:

（3）我承认“安乐死”的最重要的原因是要求“安乐死”的人的痛苦太极了。

(4) 在文明世界的今天, 男女平等, 夫妇两人一起去上班也无所谓, 只要把后脚跟的事 情处理得妥当, 那样两人的管齐下, 多增加收入, 那实在好得不过了。

例（3）在“极”与“痛苦”之间加入了程度副词“太”, 形成偏误。例（4）在粘合式程度补 语“不过”与“好”之间误加了“得”。

4.1.3 在程度补语前误加程度副词

程度补语均已虚化, 不能再受其他成分限定与修饰。例如:

（5）但汉城离我家远得很多, 我们三个不得已离开你们的怀里。

例（5）在程度补语“多”之前误加了程度副词“很”, 形成偏误。

\section{2 误用}

4.2.1 在无程度的动词后带上程度补语

(6) 因为我们的科学也发展多了。

（7）据说, 现代人比过去的人精神上过敏多了, 因为现代生活里到处都是刺激神精的。

(8) 今日的医学技术进步得很。

上述三例中的动词“发展、过敏、进步”都没有程度义，不能受程度副词修饰，也不能带 程度补语。

\section{2 .2 将绝对程度补语用于比较句中}

绝对程度补语是不含比较义, 不能用于比较句中, 能用于比较句中的程度补语只有相对 程度补语“多”。例如:

（9）这样，我比其他研究生忙得很。

（10）这几年来, 世界上的离婚率不断地增加, 以前是欧美各国的离婚率比亚洲国家多 得很,不过最近亚洲国家的离婚率也大幅度地增加。

例（9）（10）均应使用相对程度补语，此处误用了绝对程度补语“很”，应改为“我比其 他研究生忙得多”“欧美各国的离婚率比亚洲国家多得多”“我们家比别的同学家穷得多”。

4.2.3 误用程度补语共现词

有些程度补语要求有与之共现的词语，如“不过”经常与“再、最”同现，形成“再/最......不 过”格式。但学生对这类共现词的掌握不准确, 就形成如下偏误:

（11）现在市政府制定了规定，不可以公共场所边走边吸烟，真是太好不过了。

例（11）误用了“不过”的共现词，将“再”误为“太”。

4.2.4 不需用程度补语时误用

(12) 而且有两个人, 他们会互相关心得很。

（13）有一天下午, 我从学校回到家, 一开门就看父母的气色不好坏了。

例（12）出现了程度补语“很”, 根据此句的意思, 不需要用程度补语, 应改为“他们会互 相关心”。例（13）不需用程度补语“坏”，表达为“一开门就看父母的气色不好”即可。

\subsection{5 搭配词语误用}

（14）现在, 我是一名教育工作者, 每当看到小小的孩子们, 在开学的第一天, 常见到 我童年的情形, 感触深感得很啊!

例（14）应为“感触深得很”, “深感”不能带程度补语。 


\section{3 遗漏}

组合式程度补语不能直接用在述语之后，必须由助词“得”系联。例如：

（15）东京有这么多的人口，大部分的政府机关、企业总公司集中在市区中心，因此， 许多人每天要坐火车, 地铁上班, 上下班时火车内, 客满不得了, 满得比北京的公共汽车多。

例（15）中的程度补语“不得了”是组合式程度补语，应有“得”系联，此例遗漏了“得”, 形成偏误。

\section{5. 结束语}

基于上述留学生程度补语使用情况和偏误分析结果, 结合述语及其程度补语搭配的特征 与规律, 我们可考察程度补语在国内使用的对外汉语教材以及海外使用的汉语教材两类教材 中出现的情况, 基于偏误分析和教材考察结果, 结合述语及其程度补语搭配的特征与规律, 提出了关于程度补语教学及教材相关语法点编写的针对性建议。此外, 可基于上述研究, 探 索将偏误分析与搭配规律、搭配资源结合起来辅助汉语教学的研究方法, 进一步构建程度补 语与述语搭配库以及按难易程度分级的搭配的例句库, 辅助汉语教材语法点的编写、汉语学 习词典编撰和辅助汉语学习。

\section{致谢}

本文为国家社科基金一般项目《海外华语语言生活状况调查及多媒体语言资源库建设》 (13BYY090)的阶段性成果之一。

\section{References}

[1] The Language Class of Grade 1955-1957 of Chinese Department of Peking University . Explanation of Function Words in Modern Chinese ,The Commercial Press, 1982.

[2] Shao Jingmin.On the principle of semantic mutual option in Chinese Grammar. Journal of Chinese Linguistics ( the 8th issue ), The Commercial Press,1997.

[3] Zhang Yisheng. Exploration of modern Chinese adverbs, Academia Press. 2004.

[4] Cai Li, A study on the degree complement based on computational linguistics, Lecture Notes in Electrical Engineering,vol.137,pp. 733-740, 2011.

[5] Cai Li, A study on the "hen shi" structure and other issues based on calculation of large-scale corpus, Advances in Education Research(AER),vol.48,pp.8 -13, 2013.

[6] Cai Li,Range and category of degree complements in modern Chinese,Journal of Ningxia University(Humanities and Social Sciences Edition),vol.33,pp.9 -14/32,2011. 